\title{
ENERGY MECHANISMS OF FREE VIBRATIONS AND RESONANCE IN ELASTIC BODIES
}

\author{
Yu. A. Alyushin \\ National University of Science and Technology MISIS, Moscow, 119991, Russia \\ alyushin7@gmail.com; alyushin@misis.ru. Author ID in ORCID http://orcid.org/0000-0001-9977-3342. Tel.: +7 9162218591
}

\begin{abstract}
Annotation. The mechanisms of natural oscillations and resonance are described, considering the peculiarities of the transformation of elastic and kinetic energy in the implementation of the law of conservation of energy in local and integral volumes of the body, using the concept of mechanics based on the concepts of space, time and energy. When describing the motion in the Lagrange form, the elastic deformation energy of the particles is determined by the quadratic invariant of the tensor, whose components are the partial derivatives of Euler variables with respect to Lagrange variables. The increment of the invariant due to elastic deformation is represented as the sum of two scalars, one of which depends on the average value of the relative lengths of the edges of the particles in the form of an infinitesimal parallelepiped, the second is equal to the standard deviation of these lengths from the average value. It is shown that each of the scalars can be represented in the form of two dimensionless kinematic parameters of elastic energy, which participate in different ways in the implementation of the law of conservation of energy. One part of the elastic energy passes into kinetic energy and participates in the implementation of the law of conservation of energy for the body as a whole, considering external forces. The second part is not converted into kinetic energy but changes the deformed state of the particles in accordance with the equations of motion while maintaining the same level of the part of the elastic energy of the particles used for this. The kinematic parameters differ from the volume density of the corresponding types of energy by a factor equal to the elastic modulus, which is directly proportional to the density and heat capacity of the material and inversely proportional to the volume compression coefficient. Transverse, torsional, and longitudinal vibrations are considered free and under resonance conditions. The mechanisms of transformation of forced vibrations into their own after the termination of external influences and resonance at the superposition of free and forced vibrations with the same or similar frequency are considered. The formation of a new free wave at each cycle with an increase in the amplitude, which occurs mainly due to internal energy sources, and not external forces, is justified.
\end{abstract}

Keywords: equations of motion, Lagrange variables, invariants, energy model of mechanics, superposition principle, kinematic parameters of energy, free oscillations, resonance.

\section{Introduction}

Vibrations are among the most common processes, and no phenomenon in nature or any of the created mechanisms can do without them. They should be considered when calculating, manufacturing and operating building structures, transport systems, and technological processes in mechanical engineering [1-7]. The most interesting and important for practical applications include free vibrations and resonance, which are used in physics, chemistry, biology, and engineering [5], including for measuring the elastic properties of materials [6] and as an energy source [1,7].

Many papers have been devoted to the study of vibrations [3-5]. The analysis is usually limited to the shape, frequency, and period of vibrations. As a rule, vibrations of material points are considered; for elastic bodies, the relations of the elasticity theory are used, but without analyzing the energy state of particles in the body volume $[1,5]$. The Nature of free vibrations and resonance is still not fully understood. There are reasons to believe that the energy basis of free vibrations and resonance, along with the energy of external forces, are internal energy sources [8].

Free (natural) vibrations are called under the action of internal forces when the system is taken out of equilibrium. They occur at the expense of the initially reported energy from external sources without additional revenues to continue fluctuations. The resonance leads to a significant increase in 
the amplitude when forced and free vibrations interact in an elastic body with the same or similar frequency [1-5].

The purpose of the work is to describe the mechanism of natural oscillations and resonance, considering the peculiarities of the transformation of elastic and kinetic energy in the implementation of the law of conservation of energy in local and integral volumes of the body, using the concept of mechanics based on the concepts of space, time, and energy [9-10].

\section{Fundamentals of the energy model of mechanics}

The energy model of mechanics [10-11] provides a description of the motion of material particles in the form of Lagrange

$$
x_{i}=x_{i}\left(\alpha_{p}, t\right)
$$

where $t$ is time, $x_{i} \in(x, y, z)$ - current coordinates (Euler), $\alpha_{p} \in(\alpha, \beta, \gamma)$ - Lagrange variables uniquely associated with the initial coordinates of the particles. They are the arguments of all the equations used in the future. Partial derivatives $x_{i, p} \equiv \partial x_{i} / \partial \alpha_{p}$ of Euler variables $x_{i} \in(x, y, z)$ with respect to Lagrange variables $\alpha_{p} \in(\alpha, \beta, \gamma)$ forming the Jacobi matrix of transformation (1)

$$
x_{i, \alpha_{p}} \equiv \frac{\partial x_{i}}{\partial \alpha_{p}}=\left(\begin{array}{ccc}
x_{\alpha} & x_{\beta} & x_{\gamma} \\
y_{\alpha} & y_{\beta} & y_{\gamma} \\
z_{\alpha} & z_{\beta} & z_{\gamma}
\end{array}\right),
$$

together with the time derivatives $d x_{i} / d t \equiv x_{i, t}$ and $d^{2} x_{i} / d t^{2} \equiv x_{i, t t}$, the kinematic characteristics of motion are determined, including dimensionless parameters that characterize the parts of elastic energy that play a different role in the implementation of the conservation law in different volumes of the body, in particular under free vibrations [8] and resonance [12].

The different nature of the arguments in equations (1) leads to the need to use two independent infinitesimal operators: the operator $d$ for infinitesimal increments in time, for example $d x_{i}=x_{i, t} d t$ and $d x_{i, t}=x_{i, t t} d t$-increments of coordinates and velocity components in the directions of the axes $x_{i}$, the operator $\delta$ - for increments in space, for example, the volume of a particle $\delta V_{0}=\delta \alpha \delta \beta \delta \gamma$. When used simultaneously, the sequence of their writing can be changed $d \delta E=\delta d E$.

Any movement must comply with the law of conservation of energy. As a generalized scalar function [13], the energy of a moving particle $\delta E_{\text {mov }}$ must consider the independent invariants $\xi_{i}$ of equations (1). As shown in [10-11], the energy $\delta E_{\text {mov }}$ can be represented as the sum of the terms $\delta E_{i}=k_{i} \xi_{i} \delta V_{0}$ associated with each of the invariants $\xi_{i}$, in which the coefficients $k_{i}$ ensure equality of their dimensions 


$$
\delta E_{\text {mov }}=\sum_{i=1}^{n} \delta E_{i}\left(k_{i} \xi_{i}\right)=\delta V_{0} \sum_{i=1}^{n}\left(k_{i} \xi_{i}\right) .
$$

In particular, the invariant $v^{2}$ on the right side of equation (3) determines the kinetic energy of a particle with a multiplier $\left(\rho_{0} / 2\right)$

$$
\delta E_{k}=\delta m\left(v^{2}\right) / 2=\rho_{0} \delta V_{0}\left(x_{t}^{2}+y_{t}^{2}+z_{t}^{2}\right) / 2 .
$$

The increment of the energy of the external forces $\mathbf{P}$ when they move to $\mathrm{d} \mathbf{r}$

$$
d \delta E_{\mathrm{ext}}=\sum(\delta \mathbf{P} \cdot d \mathbf{r})=\sum(\delta \mathbf{P} \cdot \mathbf{v}) d t
$$

for a particle with face dimensions $\delta \alpha, \delta \beta, \delta \gamma$ for the force density

$$
\tau_{\alpha i}=\frac{\delta P_{\alpha i}}{\delta \beta \delta \gamma}, \quad \tau_{\beta i}=\frac{\delta P_{\beta i}}{\delta \alpha \delta \gamma}, \quad \tau_{\gamma i}=\frac{\delta P_{\gamma i}}{\delta \alpha \delta \beta}
$$

is [10-11]

$$
d \delta E_{e x t}=\delta V_{0}\left(\tau_{p i} x_{i, t \alpha_{p}}+x_{i, t} \frac{\partial \tau_{p i}}{\partial \alpha_{p}}\right) d t .
$$

Considering (3) - (5) when the kinetic energy changes (4),

$$
d \delta E_{k}=\delta m d\left(v^{2}\right) / 2=\rho_{0} \delta V_{0}\left(x_{t} x_{t t}+y_{t} y_{t t}+z_{t} z_{t t}\right) d t
$$

the law of conservation of energy takes the form

$$
\delta V_{0}\left[k_{1} \xi_{1, t}+k_{2} \xi_{2, t}+k_{3} \xi_{3, t}+\ldots+\tau_{p i} x_{i, t \alpha_{p}}+x_{i, t}\left(\partial \tau_{p i} / \partial \alpha_{p}-\rho_{0} x_{i, t t}\right)\right] d t=0 .
$$

Because the condition (6) must be performed in any frame of reference velocity, movement (1) must satisfy the differential equation [9-11]

$$
x_{i, t}\left(\frac{\partial \tau_{p i}}{\partial \alpha_{p}}-\rho_{0} x_{i, t t}\right)=0,
$$

where $\rho_{0}$ is the density of the material in the initial state, $\tau_{p i}$ - surface density of forces on the faces of the infinitesimal parallelepiped, the normal to which in the initial state specifies the first subscript $p \in(\alpha, \beta, \gamma)$, and the direction of the voltage - second index $i \in(x, y, z)$. In essence, these are the Kirchhoff stresses for the space of Lagrange variables [10].

If we equate each bracket to zero (this assumption may lead to the loss of some possible solutions), we obtain analogs of the differential equations of motion of classical mechanics of a deformable solid [1-3]

$$
\frac{\partial \tau_{p i}}{\partial \alpha_{p}}-\rho_{0} x_{i, t t}=0
$$

which in the absence of accelerations $\left(x_{i, t t}=0\right)$ are transformed into differential equations of equilibrium

$$
\frac{\partial \tau_{p i}}{\partial \alpha_{\mathrm{p}}}=0
$$


The energy model of mechanics allows for a transition to a single modulus of elasticity and a new scale of average stresses, considered as the volume energy density of particles [14-15]. In this case, the concept of stresses $\tau_{p i}$ becomes redundant, since they differ from the components of the tensor (2) only by a constant factor equal to twice the elastic modulus $\kappa$

$$
\tau_{p i}=2 \boldsymbol{\kappa} x_{i, p} \text {. }
$$

The modulus of elasticity $\boldsymbol{\kappa}$ is directly proportional to the density $\rho_{0}$ and the heat capacity $c$ of the material, and inversely proportional to the volume compressibility $\beta=3 \alpha$ ( $\alpha$ is the coefficient of linear expansion)

$$
\boldsymbol{\kappa}=\rho_{0} c / \beta=\rho_{0} c /(3 \alpha) .
$$

It is these physical properties that determine the behavior of materials in the region of reversible deformations [16]. Considering (8), instead of equation (7), we get

$$
x_{t}\left(\frac{\partial x_{p}}{\partial \alpha_{p}}-\mu^{2} x_{t t}\right)+y_{t}\left(\frac{\partial y_{p}}{\partial \alpha_{p}}-\mu^{2} y_{t t}\right)+z_{t}\left(\frac{\partial z_{p}}{\partial \alpha_{p}}-\mu^{2} z_{t t}\right)=0,
$$

where $\mu^{2}=\rho_{0} /(2 \kappa)$. If in (10) we equate each bracket to zero, the differential equations of motion are transformed into Poisson equations for each of the functions (1)

$$
\partial^{2} x_{i} / \partial \alpha_{p}^{2}=\mu^{2}\left(\partial^{2} x_{i} / \partial t^{2}\right)
$$

Solutions of any types of problems based on the energy model of mechanics with a single modulus of elasticity [10-11] do not contradict those known from classical mechanics. This model leads to a significant reduction in mathematical difficulties and will be used in the future to describe the mechanism of free oscillations and resonance.

To achieve the goal set in this paper, the quadratic invariant of the matrix (2), which is equal to the sum of the squares of its elements,

To achieve the goal set in this paper, particular importance the quadratic invariant of the matrix (2), which is equal to the sum of the squares of its elements,

$$
\Gamma_{e}^{2}=x_{\alpha}^{2}+y_{\alpha}^{2}+z_{\alpha}^{2}+x_{\beta}^{2}+y_{\beta}^{2}+z_{\beta}^{2}+x_{\gamma}^{2}+y_{\gamma}^{2}+z_{\gamma}^{2} .
$$

It determines the volume density of elastic energy $\delta E_{e}$ considering the initial state of the particles [10-11]

$$
\delta E_{e} / \delta V_{0}=\kappa \Gamma_{e}^{2}
$$

In the future, we will use dimensionless kinematic parameters of the volume density of the corresponding types of energy by type $e_{i}=\delta E_{i} /\left(\kappa \delta V_{0}\right)$. The invariant (12) is a kinematic parameter of the volume density of the elastic energy of the particles

$$
\Gamma_{e}^{2}=\delta E_{e} /\left(\kappa \delta V_{0}\right) \text {. }
$$


The energy of the particle gained $\left(e_{d e f}>0\right)$ or lost $\left(e_{d e f}<0\right)$ due to the reduction of elastic deformation $\delta E_{d e f}=\kappa e_{\text {def }} \delta V_{0}$, in comparison with the initial state, determines the local kinematic parameter

$$
e_{d e f}=\Gamma_{e}^{2}-3
$$

The right part in equation (12) can be written in terms of the squares of the ratios of the lengths of the edges up to $\delta l_{0}$ and after the deformation $\delta l$, initially oriented in the direction of the corresponding axes

$$
l_{p}^{2}=\left(\delta l / \delta l_{0}\right)_{p}^{2}=x_{p}^{2}+y_{p}^{2}+z_{p}^{2}, \quad p \in(\alpha, \beta, \gamma) .
$$

Then parameter (14) can be represented in terms of other dimensionless scalars $e_{e}$ and $e_{s}$

$$
\begin{gathered}
e_{d e f}=\Gamma_{e}^{2}-3=3\left(e^{2}-1\right)+e_{s}=e_{e}+e_{s}, \\
e=\left(l_{\alpha}+l_{\beta}+l_{\gamma}\right) / 3, \quad e_{e}=3\left(e^{2}-1\right), \quad e_{s}=\left(l_{\alpha}-e\right)^{2}+\left(l_{\beta}-e\right)^{2}+\left(l_{\gamma}-e\right)^{2},
\end{gathered}
$$

where $e$ is the average of the relative lengths of the edges $l_{p}$ of an infinitesimal parallelepiped before and after the deformation; $e_{e}$ depends on the average length $e$, can be either positive or negative. The value of $e_{s}$ is always positive and coincides with the standard deviation of the lengths of the edges of the parallelepiped $l_{p}$ from their average value $e$. Equation (15) allows for a change in the deformed state of the particles due to internal energy sources, if $e_{e}+e_{s}=$ const.

For the oscillations considered below, the values of $e_{e}$ and $e_{s}$ can be represented in terms of additional kinematic parameters of the type $e_{e}=e_{e 1}+e_{e 2}$ and $e_{s}=e_{s 1}+e_{s 2}$, which play a different role in fulfilling the law of conservation of energy for particles and the body as a whole. In particular, the parameters $e_{e l}$ and $e_{s l}$ will include the fractions of energy that are involved in the transformation of the kinetic energy of particles into elastic or vice versa, as well as in the implementation of the law of conservation of energy for an elastic body as a whole, considering external forces. The parameters $e_{e 2}$ and $e_{s 2}$ consider the change only in elastic deformation within the particles, for example, the transition of the energy of volume change to the energy of shape change or vice versa. They do not require an influx of energy through its boundaries, ensure the implementation of the law of conservation of energy in the volumes of particles and do not affect the change in the energy of elastic deformation of the body.

Considering the observations made and the initial state $\left(\Gamma_{e}^{2}=3\right)$, the energy of the particle $\delta E_{d e f}$ with a volume of $\delta V_{0}$, acquired due to deformation, can be represented as [9-11]

$$
\delta E_{d e f}=\kappa \delta V_{0}\left(\Gamma_{e}^{2}-3\right)=\kappa \delta V_{0}\left[3\left(e^{2}-1\right)+e_{s}\right]=\kappa \delta V_{0}\left(e_{e}+e_{s}\right)=\kappa \delta V_{0}\left(e_{e 1}+e_{e 2}+e_{s 1}+e_{s 2}\right)
$$

The ratio of the particle volumes after $\delta V$ and before the deformation $\delta V_{0}$ determines the Jacobian of the transformation (1) or the cubic invariant of the tensor (2) 


$$
R=\delta V / \delta V_{0}=\left|\begin{array}{lll}
x_{\alpha} & x_{\beta} & x_{\gamma} \\
y_{\alpha} & y_{\beta} & y_{\gamma} \\
z_{\alpha} & z_{\beta} & z_{\gamma}
\end{array}\right| .
$$

In all the cases discussed below, an elastic rod of length $L$ with a cross-section $S_{0}$ is considered as a physical body, the ends of which are fixed in fixed arrays that do not exchange energy with the oscillating system.

For the energy justification of the resonance, the transformations of forced vibrations into free ones after the termination of the action of external forces, as well as the subsequent occurrence of the resonance when periodic external forces appear with the frequency of natural vibrations or close to it, are considered.

\section{Transverse vibrations}

Consider the energy features of transverse vibrations in accordance with the equations

$$
x\left(\alpha_{p}, t\right)=\alpha, \quad y\left(\alpha_{p}, t\right)=\beta+v(\alpha, t), \quad z\left(\alpha_{p}, t\right)=\gamma,
$$

where $v(\alpha, t)$ - moves in the direction of the $y$-axis. We start counting the time when there is no deformation $v(\alpha, \mathrm{t})=0$ and the Lagrangian coordinates coincide with the initial ones. The vibrations allowed by the law of conservation of energy must correspond to equation (10), which is converted to the form

$$
y_{t t}(\alpha, t)=\left(2 \kappa / \rho_{0}\right) y_{\alpha \alpha} .
$$

This equation under the initial condition $\alpha_{i}=x_{i}\left(\alpha_{p}, t=0\right)$ and boundary conditions for displacements $v(\alpha=0, \mathrm{t})=0, v(\alpha=L, \mathrm{t})=0$, is fulfill by the function

$$
y(\alpha, t)=\beta+q \sin (\pi \alpha / L) \sin \left(\omega_{0} t\right),
$$

with derivatives

$$
y_{\alpha \alpha}(\alpha, t)=-q\left(\frac{\pi}{L}\right)^{2} \sin \left(\pi \frac{\alpha}{L}\right) \sin \left(\omega_{0} t\right), \quad y_{t t}(\alpha, t)=-q \omega^{2} \sin \left(\pi \frac{\alpha}{L}\right) \sin \left(\omega_{0} t\right),
$$

where $q$ is the maximum displacement of particles along the y axis in the cross section $\alpha=L / 2$.

Natural frequency

$$
\omega_{0}=(\pi / L) \sqrt{2 \kappa / \rho_{0}}
$$

considers the properties of the material and the size of the sample. Velocities of particles

$$
y_{t}(\alpha, t)=v_{t}(\alpha, t)=q \omega_{0} \sin (\pi \alpha / L) \cos \left(\omega_{0} t\right)
$$

at the ends of the rod are equal to 0 , at $t=\pi n / \omega_{0}$ they are maximal in each of the sections along its entire length. Considering (15) the tensor 


$$
x_{i, p}=\left(\begin{array}{ccc}
1 & 0 & 0 \\
q \pi / L \cos (\pi \alpha / L) \sin \left(\omega_{0} t\right) & 1 & 0 \\
0 & 0 & 1
\end{array}\right)
$$

corresponds to the system (19).

The deformation is carried out due to shifts $\partial y / \partial \alpha \equiv y_{\alpha}$. The quadratic invariant of the tensor determines the increment of the local energy $\delta E_{d e f}$ and power $\delta W_{d e f}=d \delta E_{d e f} / d t$ of the elastic deformation of the particles

$$
\begin{gathered}
\delta E_{d e f}=\kappa\left(\Gamma_{e}^{2}-3\right) \delta V_{0}=\kappa \delta V_{0}(\pi q / L)^{2} \cos ^{2}(\pi \alpha / L) \sin ^{2}\left(\omega_{0} t\right), \\
\delta W_{d e f} /\left(2 \kappa \delta V_{0}\right)=\omega_{0}(\pi q / L)^{2} \cos ^{2}(\pi \alpha / L) \sin \left(\omega_{0} t\right) \cos \left(\omega_{0} t\right) .
\end{gathered}
$$

The energy for deformation in the volume of the rod is

$$
E_{d e f}=\frac{1}{2} \kappa V_{0}(\pi q / L)^{2} \sin ^{2}\left(\omega_{0} t\right)
$$

For kinetic energy with velocity (24)

$$
\delta E_{k i n}=\frac{1}{2} \rho_{0} v_{t}^{2} \delta V_{0}=\frac{1}{2} \delta V_{0} \rho_{0}\left[q \omega_{0} \sin (\pi \alpha / L) \cos \left(\omega_{0} t\right)\right]^{2}
$$

after integration by volume, we get

$$
E_{k i n}=\frac{L}{4} S_{0} \rho_{0} q^{2} \omega_{0}^{2} \cos ^{2}\left(\omega_{0} t\right)=0,5 V_{0} \kappa(\pi q / L)^{2} \cos ^{2}\left(\omega_{0} t\right)
$$

Total kinetic energy and strain energy in the volume of the oscillating rod

$$
E_{\text {sum }}=E_{\text {def }}+E_{\text {kin }}=S_{0} \kappa \frac{\pi^{2} q^{2}}{2 L}\left[\sin ^{2}\left(\omega_{0} t\right)+\cos ^{2}\left(\omega_{0} t\right)\right]=\frac{1}{2} V_{0} \kappa\left(\frac{\pi q}{L}\right)^{2}=\text { const }
$$

coincides with the kinetic energy available in the system (28) at the moment $t=0$ and does not change in time, which indicates compliance with the law of conservation of energy, if there is no energy transfer to the fixed walls at the contacts with the ends of the sample or to the environment from the outer surface of the rod.

Condition (23) corresponds to proper transverse vibrations in an elastic rod without energy consumption from external forces and without changing the volume of particles

$$
R=\delta V / \delta V_{0}=\left|\begin{array}{ccc}
1 & 0 & 0 \\
y_{\alpha} & 1 & 0 \\
0 & 0 & 1
\end{array}\right|=1
$$

At the end of the cycle $\left(t=T=2 \pi / \omega_{0}\right)$, the system returns to its initial state, the elastic energy is absent, and the kinetic energy (22) takes the maximum possible value. 
To find out the energy features of the resonance, we consider vibrations with a driving force $[1-2]$

$$
F=F_{0} \sin (\omega t)
$$

acting in the Central section along the length $\alpha=L / 2$ with an amplitude $F_{0}$ and a circular frequency $\omega$, which does not necessarily coincide with $\omega_{0}$. The force $F_{0}$ can be determined from the integral law of conservation of energy in the form of equality of the power of the external force and the rate of change of kinetic and elastic energy in the volume of the rod.

Considering the velocity $y_{t}$ in the cross section $\alpha=L / 2$, where the force (30) is applied, we obtain the power

$$
\mathrm{W}_{e x t}=\left.F y_{t}\right|_{\alpha=L / 2}=F_{0} q \omega \sin (\omega t) \cos (\omega t) .
$$

The energy transferred to the system is converted into elastic (19) and kinetic (21) energy particles of the rod, which characterize the specific powers $\delta W_{k i n}$ and $\delta W_{d e f}$

$$
\begin{gathered}
\delta W_{k i n}=d \delta E_{k i n} / d t=0,5 \rho_{0} \delta V_{0} d y_{t}^{2} / d t=2 \kappa \delta V_{0} y_{t} y_{t t} / \lambda_{0}^{2}, \\
\frac{\delta W_{d e f}}{2 \kappa \delta V_{0}}=y_{\alpha} y_{t \alpha}=\omega(q \pi / L)^{2} \cos ^{2}(\pi \alpha / L) \sin (\omega t) \cos (\omega t) .
\end{gathered}
$$

In the expression for the rate of change of the kinetic energy of the particle $\delta W_{k i n}$, the material constant is used $\lambda_{0}^{2}=2 \kappa / \rho_{0}$, which determines the circular frequency (23). The ratio $\eta=\omega / \omega_{0}$ characterizes both the kinematic features of vibrations $(\omega)$ and the physical properties of the material $\left(\omega_{0}\right)$. Considering the derivatives (22) and (24) of the function (21) in time and Lagrange variables at the force frequency $\omega$, the local powers of the kinetic $\delta W_{k i n}$ and elastic $\delta W_{d e f}$ particle energy is determined by the equations

$$
\begin{gathered}
\frac{\delta W_{k i n}}{2 \kappa \delta V_{0}}=-\omega(q \pi \eta / L)^{2} \sin ^{2}(\pi \alpha / L) \sin (\omega t) \cos (\omega t) \\
\frac{\delta W_{d e f}}{2 \kappa \delta V_{0}}=\omega(q \pi / L)^{2} \cos ^{2}(\pi \alpha / L) \sin (\omega t) \cos (\omega t)
\end{gathered}
$$

The total power integral in the rod volume at any given time will be

$$
\frac{W_{s}}{2 \kappa V_{0}}=\frac{W_{d e f}}{2 \kappa V_{0}}+\frac{W_{k i n}}{2 \kappa V_{0}}=0,5 \omega(q \pi / L)^{2}\left(1-\eta^{2}\right) \sin (\omega t) \cos (\omega t) \text {. }
$$

Note that when describing the motion in the Lagrange form, it is not necessary to monitor the change in the contour of an elastic body during the oscillation, since for a Lagrangian coordinate system, it coincides with the original configuration and does not change in time. 
Equating the powers of external (31) and internal (33) forces, we find the force $F_{0}$ corresponding to the vibrations with the frequency and amplitude considered

$$
F_{0}=\kappa V_{0} q\left(1-\eta^{2}\right)(\pi / L)^{2}
$$

Depending on the frequency ratio, the force $F_{0}$ can be positive $\left(\omega<\omega_{0}\right)$, negative ( $\left.\omega>\omega_{0}\right)$, and zero $\left(\omega=\omega_{0}\right)$. the Positive force, as follows from equation (31), supplies energy to the rod in the first and third quarters of the cycle, when the kinetic energy is converted into elastic deformation of the particles. In the other two quarters, the power will be negative, the energy of elastic deformation of the particles is converted into kinetic energy and transmitted to an external source. A negative force $F_{0}$ occurs when the circular frequency of the periodic external force exceeds the natural frequency of the system. Then, in the first and third quarters of the cycle, the power is negative, and the kinetic energy is spent on the deformation of the particles and transferred to an external source.

Equations (30), (31) - (33) correspond to forced vibrations in an elastic rod with a period $T=$ $2 \pi / \omega$ under the action of an exciting force (34). After the cycle is completed, the system returns to its original state and, if the force (30) continues to operate, the cycle repeats.

If the external force ceases after the next cycle, regardless of the frequency ratio, the kinetic energy of the particles remains in the system

$$
\left.\delta E_{k i n}\right|_{t=T}=0,5 \rho_{0} v_{t}^{2} \delta V_{0}=0,5 \delta V_{0} \rho_{0}[q \omega \sin (\pi \alpha / L)]^{2}=\kappa \delta V_{0}(\pi \eta q / L)^{2} \sin ^{2}(\pi \alpha / L),
$$

which will lead to continued fluctuations. The frequency and amplitude may vary, but equations (32) - (33) remain valid.

It follows from (3) that if the frequency of the external force is less than own $\omega<\omega_{0}$, then the positive power $W_{s}>0$ in the first quarter of the cycle will increase the actual frequency $\omega$. Similarly, when $\omega>\omega_{0}$ the negative power of $W_{s}<0$ will reduce the actual frequency. Only in the case that $\omega=\omega_{0}$ the volume integral power is equal to 0 over the entire cycle, the sum of kinetic and elastic energy in the system remains unchanged, which corresponds to the definition of free vibrations that can continue without energy input from external sources [1-5].

From the point of view of resonance, the case is interesting when a cyclic force (30) acts, creating a forced oscillation with a frequency of natural vibrations $\omega=\omega_{0}$ or close to it. Then the two waves will interact, forming a new wave.

In accordance with the superposition principle $[11,17]$, to obtain the equations of joint motion, it is sufficient to replace the Lagrange variables of external (superimposed) motion with expressions for the corresponding Euler variables of internal (nested) motion. In our case, the equations for natural and forced oscillations may differ in the circular frequency $\omega$ and the amplitude $q$, but they are 
equivalent in their effect on the resulting oscillation. Any of them can be considered external, the other internal.

We choose the amplitude of natural oscillations by the lower index $q_{0}$

$$
x\left(\alpha_{p}, t\right)=\alpha, \quad y(\alpha, t)=\beta+q_{0} \sin (\pi \alpha / L) \sin \left(\omega_{0} t\right), \quad z\left(\alpha_{p}, t\right)=\gamma .
$$

For a forced oscillation taking into account the resonance, we use the equation

$$
x\left(\alpha_{p}, t\right)=\alpha, \quad y(\alpha, t)=\beta+q_{1} \sin (\pi \alpha / L) \sin \left(\omega_{0} t\right), \quad z\left(\alpha_{p}, t\right)=\gamma .
$$

Replacing the variable $\beta$ in the system (35) with the right part of the equations (36), for the joint motion we obtain

$$
x\left(\alpha_{p}, t\right)=\alpha, \quad y(\alpha, t)=\beta+\left(q_{0}+q_{1}\right) \sin (\pi \alpha / L) \sin \left(\omega_{0} t\right), \quad z\left(\alpha_{p}, t\right)=\gamma .
$$

If the frequencies of external and natural oscillations on the new cycle are equal, the amplitude will be equal to the sum of the amplitudes of forced (on the current cycle) and natural (on the previous cycle) oscillations of the system. To clarify the question of the energy possibility of such oscillations, it is necessary to additionally determine the kinetic and elastic energies using derivatives of equations

$$
y_{t}=\left(q_{0}+q_{1}\right) \omega_{0} \sin (\pi \alpha / L) \cos \left(\omega_{0} t\right), \quad y_{\alpha}=\left(q_{0}+q_{1}\right)(\pi / \mathrm{L}) \cos (\pi \alpha / L) \sin \left(\omega_{0} t\right) .
$$

The integral values are equal to

$$
E_{\text {def }}=0,5 \kappa V_{0}\left(q_{0}+q_{1}\right)^{2}(\pi / L)^{2} \sin ^{2}\left(\omega_{0} t\right), \quad E_{k i n}=0,5 \kappa V_{0}\left(q_{0}+q_{1}\right)^{2}(\pi \eta / L)^{2} \cos ^{2}\left(\omega_{0} t\right) .
$$

Only these equations, by analogy with (26), (28), can ensure the continuation of oscillations with the fulfillment of the law of conservation of energy for the system as a whole

$$
E_{d e f}+E_{k i n}=0,5 \kappa V_{0}\left(q_{0}+q_{1}\right)^{2}(\pi / L)^{2}=\text { const } .
$$

As a result, we get a new free oscillation with an increased amplitude, which can interact with a new cycle of forced oscillation. An increase in the amplitude of free vibrations due to interaction with forced vibrations with the frequency of natural vibrations or close to it is the basis of resonance [12]. Equation (38) can be considered the energy justification of the resonance. The kinetic and elastic energies in the body volume increase in proportion to the square of the new amplitude due to internal sources determined by the elastic modulus of the material.

The superposition principle [17] and the new model of mechanics with a single elastic modulus (8) confirm the kinematic and energetic possibility of implementing joint motion (37) in compliance with the law of conservation of energy. At equal amplitudes $q_{0}=q_{1}$, all energy characteristics of the combined oscillation increase by 4 times in relation to the initial free oscillation.

To identify the role of internal energy sources, we pay attention to the kinematic parameters of the energy invariants (15) - (16), which depend on the relative average length e and standard 
deviations es. For each particle, you can point out specific 4 fractions of the dimensionless bulk energy density

$$
\begin{gathered}
e_{e}=e_{e 1}+e_{e 2}=+\frac{1}{3}\left(\frac{\pi q}{L}\right)^{2} \cos ^{2}\left(\frac{\pi \alpha}{L}\right) \sin ^{2}\left(\omega_{0} t\right)+\frac{4}{3}\left\{\left[1+\left(\frac{\pi q}{L}\right)^{2} \cos ^{2}\left(\frac{\pi \alpha}{L}\right) \sin ^{2}\left(\omega_{0} t\right)\right]^{1 / 2}-1\right\}, \\
e_{s}=e_{s 1}+e_{s 2}=\frac{2}{3}\left(\frac{\pi q}{L}\right)^{2} \cos ^{2}\left(\pi \frac{\alpha}{L}\right) \sin ^{2}\left(\omega_{0} t\right)-\frac{4}{3}\left\{\left[1+\left(\frac{\pi q}{L}\right)^{2} \cos ^{2}\left(\pi \frac{\alpha}{L}\right) \sin ^{2}\left(\omega_{0} t\right)\right]^{1 / 2}-1\right\}, \\
e_{d e f}=e_{e}+e_{s}=\left(\frac{\pi q}{L}\right)^{2} \cos ^{2}\left(\frac{\pi \alpha}{L}\right) \sin ^{2}\left(\omega_{0} t\right) .
\end{gathered}
$$

They carry objective information, including about energy sources that are not related to external forces and are not converted into kinetic energy of particles. The most informative are the relative measures with respect to the total elastic energy of the particle (14). Considering the first two terms of the function expansion in a series $\sqrt{1+x}=1+1 / 2 x-1 / 8 x^{2}+\ldots$, these relations remain the same for all particles at any given time

$$
\frac{e_{e 1}}{\mathrm{e}_{d e f}}+\frac{e_{e 2}}{\mathrm{e}_{d e f}}=\frac{1}{3}+\frac{2}{3}, \quad \frac{e_{s 1}}{e_{d e f}}+\frac{e_{s 2}}{e_{d e f}}=\frac{2}{3}-\frac{2}{3} .
$$

As follows from equations (39), the fractions of its energy $e_{e l}=1 / 3 e_{d e f}$ and $e_{s l}=2 / 3 e_{d e f}$ change synchronously and participate in the transformation of elastic energy into kinetic energy. Their amount

$$
e_{d e f}=e_{e}+e_{s}=e_{e 1}+e_{s 1}=\left(\frac{\pi q}{L}\right)^{2} \cos ^{2}\left(\frac{\pi \alpha}{L}\right) \sin ^{2}\left(\omega_{0} t\right)
$$

ensures the implementation of the law of conservation of energy in the volume of the rod with an integral volume value (26) and a phase shift of $\pi / 2$ with respect to the change in the kinetic energy of the particles (28).

The energy shares of $e_{e 2}$ and $e_{s 2}$ vary in opposite phases, the sum of these shares is always 0 , although each of them is comparable to the total energy of $e_{\text {def. }}$. In other words, the energy $e_{e 2}$, determined by the change in the average length of the edges of the particle in the form of an infinitesimal parallelepiped, passes into the energy $e_{s 2}$, associated with the standard deviation of the relative lengths of the edges of the particle from their average value, and Vice versa. Such deformations do not change the energy state of the particle and the body as a whole.

The addition of velocities in accordance with the principle of superposition of motions (37) provides the necessary kinetic energy for the deformation of particles during the cycle and the fulfillment of the law of conservation of energy for the system as a whole, considering external forces.

Further development of oscillation can occur in one of the following ways: 
1) the appearance of a new oscillation with an amplitude depending on the frequency ratio,

$$
y(\alpha, t)=\beta+\left[q_{0} \sin \left(\omega_{0} t\right)+q n \sin (\omega t)\right] \sin (\pi \alpha / L),
$$

if, after the formation of free vibrations, the external force (30) begins to act again with a frequency $\omega$ significantly different from $\omega_{0}$. The influence of the free wave will decrease and forced oscillations (36) with the frequency of force (30) will continue, which require power (31);

2) continuation of free oscillations with an increase in the amplitude $q_{0}+q_{1}+\ldots+q_{i}$ after the next superposition, if the frequency of forced oscillations is close to its own and there is no energy exchange with the environment;

3) decrease the amplitude if the resonant system is used as an accumulator energy and stored in the system energy will go into the environment, including due to displacement were considered stationary supporting walls of a perfectly rigid body, in which is fixed the elastic rod.

The most dangerous is the continuation of vibrations in the conditions of resonance with the achievement and subsequent exceeding of the limit values of the stresses acting in the system, the occurrence of irreversible deformations or destruction of the system.

\section{Torsional vibrations}

During torsional vibrations, circumferential and radial movements of particles can occur. In this regard, under the condition of plane deformation in the Cartesian coordinate system, two of the three equations (11) must be considered

$$
y_{\alpha \alpha}+y_{\beta \beta}+y_{\gamma}=\mu^{2} y_{t t}, \quad z_{\alpha \alpha}+z_{\beta \beta}+z_{\gamma \gamma}=\mu^{2} z_{t t} .
$$

If ignore the change in the radial coordinate and accept

$$
x=\alpha, \quad y=\beta \cos \Delta \psi-\gamma \sin \Delta \psi, \quad z=\beta \sin \Delta \psi+\gamma \cos \Delta \psi,
$$

equations (40) take the form

$$
\begin{aligned}
& -y \psi_{\alpha}^{2}-z \psi_{\alpha \alpha}=-\mu^{2} y \psi_{t}^{2}-\mu^{2} z \psi_{t t}, \\
& -z \psi_{\alpha}^{2}+y \psi_{\alpha \alpha}=-\mu^{2} z \psi_{t}^{2}+\mu^{2} y \psi_{t t} .
\end{aligned}
$$

Squaring and summing the left and right parts, we obtain an equation of degree 4 for the function $\psi(\alpha, t)$,

$$
\psi_{\alpha}^{4}+\psi_{\alpha \alpha}^{2}=\mu^{4} \psi_{t}^{4}+\mu^{4} \psi_{t t}^{2}
$$

The solution $\psi(\alpha, t)=C \sin (\alpha \pm q t)$ turns equation (42) into an identity, but it does not agree with the initial and boundary conditions

$$
\psi(\alpha, \mathrm{t}=0)=\theta \sin \left(\pi \frac{\alpha}{L}\right), \quad \psi(\alpha=L / 2, \mathrm{t}=0)=\theta, \quad \psi_{t}(\alpha, \mathrm{t}=0)=0,
$$




$$
\psi(\alpha=0, \mathrm{t})=0, \quad . \psi(\alpha=L, \mathrm{t})=0 .
$$

If we accept $\psi_{\alpha}^{4}=\mu^{4} \psi_{t}^{4}$, then for the function $\psi=\psi(\alpha, t)$ we obtain an equation similar to (14) for transverse vibrations,

$$
\psi_{\alpha \alpha}^{2}-\mu^{4} \psi_{t t}^{2}=0
$$

with a solution

$$
\begin{gathered}
\Delta \psi(\alpha, t)=\theta \sin (\pi \alpha / L) \sin \left(\omega_{0} t\right), \\
\omega_{0}=(\pi / L) \sqrt{2 \kappa / \rho_{0}},
\end{gathered}
$$

where $\omega_{0}$ is the frequency of natural vibrations, $\theta$ is the angle of rotation of the rod in the cross section with the coordinate $\alpha=L / 2$ at $t=\pi /\left(2 \omega_{0}\right)$. In this case, the equations (40), as well as the boundary and initial conditions (43) are fulfilled.

Time and direction derivatives

$$
\begin{array}{cc}
\psi_{t}(\alpha, t)=\theta \omega_{0} \sin (\pi \alpha / L) \cos \left(\omega_{0} t\right), & \psi_{t t}(\alpha, t)=-\theta \omega_{0}^{2} \sin (\pi \alpha / L) \sin \left(\omega_{0} t\right), \\
\psi_{\alpha}(\alpha, t)=\theta \pi / L \cos (\pi \alpha / L) \sin \left(\omega_{0} t\right), & \psi_{\alpha \alpha}(\alpha, t)=-\theta(\pi / L)^{2} \sin (\pi \alpha / L) \sin \left(\omega_{0} t\right), \\
\psi_{\alpha t}(\alpha, t)=\theta \omega_{0} \pi / L \cos (\pi \alpha / L) \cos \left(\omega_{0} t\right),
\end{array}
$$

determine the kinematic, deformation and energy characteristics of the particles and the body as a whole. Note that the obtained solution satisfies not only the system (6), but also the more general equation (5)

$$
y_{t}\left(\mathrm{y}_{\alpha \alpha}+\mathrm{y}_{\beta \beta}+\mathrm{y}_{\gamma}-\mu^{2} y_{t t}\right)+z_{t}\left(z_{\alpha \alpha}+z_{\beta \beta}+z_{\gamma \gamma}-\mu^{2} z_{t t}\right)=0
$$

In the latter case, only the condition (44) in a simplified form should be met

$$
\psi_{\alpha \alpha}-\mu^{2} \psi_{t t}=0
$$

This can be considered as an additional argument about the acceptability of the obtained solution for analyzing the energy features of free torsional vibrations.

Considering the components of the tensor (2)

$$
x_{i, p}=\left(\begin{array}{ccc}
1 & 0 & 0 \\
-\psi_{\alpha} z & \cos \Delta \psi & -\sin \Delta \psi \\
\psi_{\alpha} y & \sin \Delta \psi & \cos \Delta \psi
\end{array}\right),
$$

we find the value of the quadratic invariant, the specific energy of elastic deformation, and the kinetic energy of particles

$$
\begin{gathered}
\Gamma_{e}^{2}=l_{\alpha}^{2}+l_{\beta}^{2}+l_{\gamma}^{2}=3+y_{\alpha}^{2}+z_{\alpha}^{2}=3+\psi_{\alpha}^{2} r^{2}, \\
\delta E_{d e f}=\kappa \delta V_{0} \psi_{\alpha}^{2} r^{2}=\kappa \delta V_{0} \pi^{2} \theta^{2}(r / L)^{2} \cos ^{2}(\pi \alpha / L) \sin ^{2}\left(\omega_{0} t\right),
\end{gathered}
$$




$$
\delta E_{k i n}=\kappa \mu^{2} \delta V_{0} \psi_{t}^{2} r^{2}=\kappa \delta V_{0} \theta^{2} \pi^{2}(r / L)^{2} \sin ^{2}(\pi \alpha / L) \cos ^{2}\left(\omega_{0} t\right),
$$

which depend on the particle radius $r$. Volume-integral energy values

$$
E_{d e f}=\kappa V_{0} \pi^{2} \theta^{2} \frac{R^{2}}{4 L^{2}} \sin ^{2}\left(\omega_{0} t\right), \quad E_{k i n}=\kappa V_{0} \theta^{2} \pi^{2} \frac{R^{2}}{4 L^{2}} \cos ^{2}\left(\omega_{0} t\right),
$$

in sum, they correspond to the law of conservation of energy in the volume of the oscillating rod and coincide with the work of external forces transmitted to the body at the time of the beginning of vibrations, which corresponds to the concept of natural vibrations

$$
E_{\text {sum }}=E_{\text {def }}+E_{\text {kin }}=\kappa V_{0} \theta^{2} \pi^{2} \frac{R^{2}}{4 L^{2}}=\text { const }
$$

In accordance with equations (41) and (45), as in the case of transverse vibrations, elastic deformation is carried out due to shifts, the volume of material particles and the density of the material remain unchanged, regardless of the magnitude of the rotation angle $\psi$

$$
R=\delta V / \delta V_{0}=\left|\begin{array}{ccc}
x_{\alpha} & x_{\beta} & x_{\gamma} \\
y_{\alpha} & y_{\beta} & y_{\gamma} \\
z_{\alpha} & z_{\beta} & z_{\gamma}
\end{array}\right|=\left|\begin{array}{ccc}
1 & 0 & 0 \\
-\psi_{\alpha} z & \cos \Delta \psi & -\sin \Delta \psi \\
\psi_{\alpha} y & \sin \Delta \psi & \cos \Delta \psi
\end{array}\right|=1 .
$$

The cause of forced torsional vibrations may be the moment

$$
M=M_{0} \sin (\omega t)
$$

with an amplitude $M_{0}$, that acts in a cross section with the coordinate $\alpha=L / 2$ with a frequency $\omega$ that does not necessarily coincide with the proper $\omega_{0}$. Considering (41), (45) and angular velocity $\left.\psi_{t}\right|_{\alpha=L / 2}=\theta \omega \cos (\omega t)$, the moment (48) produces power

$$
\mathrm{W}_{e x t}=\left.M \psi_{t}\right|_{\alpha=L / 2}=M_{0} \theta \omega \sin (\omega t) \cos (\omega t),
$$

converted into elastic and kinetic energy of particles, for the rate of change of which (taking into $\left.\delta W_{i}=d \delta E_{i} / d t\right)$ the equations are valid

$$
\begin{gathered}
\frac{\delta W_{d e f}}{2 \kappa \delta V_{0}}=\psi_{\alpha} \psi_{\alpha t} r^{2}=\pi^{2} \theta_{0}^{2} \omega(r / L)^{2} \cos ^{2}(\pi \alpha / L) \sin (\omega t) \cos (\omega t) \\
\frac{\delta W_{k i n}}{2 \kappa \delta V_{0}}=\frac{1}{\lambda_{0}^{2}} \psi_{t} \psi_{t t} r^{2}=-\theta_{0}^{2} \pi^{2} \eta^{2} \omega(r / L)^{2} \sin ^{2}(\pi \alpha / L) \sin (\omega t) \cos (\omega t)
\end{gathered}
$$

As in the case of transverse vibrations, the kinetic energy of particles depends on the density of the material $\rho_{0}$, so it contains a multiplier $\eta=\omega / \omega_{0}$, which characterizes the ratio of the frequencies of forced and natural vibrations.

Integrating the powers by volume

$$
W_{d e f}=0,5 \kappa V_{0} \pi^{2} \theta^{2}(R / L)^{2} \omega \sin (\omega t) \cos (\omega t),
$$




$$
W_{k i n}=-0,5 \kappa V_{0} \theta^{2} \pi^{2} \eta^{2}(R / L)^{2} \omega \sin (\omega t) \cos (\omega t),
$$

and using the energy identity that includes the external moment (49), as well as the internal forces (50) and (51), we find the moment

$$
M_{0}=0,5 \kappa V_{0} \theta \pi^{2}(R / L)^{2}\left(1-\eta^{2}\right) .
$$

The system (40) under the action of a moment (48) with an amplitude (52) corresponds to forced harmonic oscillations with a circular frequency of the external moment $\omega$. If the external moment (48) ceases to act, for example, after the completion of the next cycle, the kinetic energy of the particles remains in the system, which causes its own vibrations.

Sum of capacities (50) and (51)

$$
W_{d e f}+W_{k i n}=0,5 \kappa V_{0} \theta_{0}^{2} \pi^{2}(R / L)^{2}\left(1-\eta^{2}\right) \omega \sin (\omega t) \cos (\omega t)
$$

in the absence of external influence, it characterizes possible transitions of elastic energy to kinetic energy and Vice versa. Stationary mode occurs when $\omega / \omega_{0}=1$. If the actual frequency of vibrations is lower than the proper $\omega_{0}$ determined by the elastic properties of the material (46), the positive power will lead to its increase by 1 and 3 quarters of cycles. Otherwise, the oscillation frequency will decrease, and the mode will correspond to its own oscillations. Equation (53) can be considered as a mechanism for converting forced oscillations into proper ones after the external influence ceases.

This feature is confirmed by the analysis of the total elastic and kinetic energy in the rod volume, which depends on the frequency ratio

$$
E_{d e f}+E_{k i n}=\kappa V_{0} \theta^{2} \pi^{2} \frac{R^{2}}{4 L^{2}}\left[\sin ^{2}(\omega t)+\eta^{2} \cos ^{2}(\omega t)\right]
$$

and only if they are equal $(\eta=1)$ remains constant, as follows from the definition of the system's natural oscillations [1-5].

Resonance is possible if the free vibrations are superimposed forced with a circular frequency of natural vibrations $\omega 0$. Let's use the equations for natural oscillations

$$
x=\alpha, \quad y=\beta \cos \Delta \psi_{0}-\gamma \sin \Delta \psi_{0}, \quad z=\beta \sin \Delta \psi_{0}+\gamma \cos \Delta \psi_{0},
$$

and forced fluctuations

$$
x=\alpha, \quad y=\beta \cos \Delta \psi_{1}-\gamma \sin \Delta \psi_{1}, \quad z=\beta \sin \Delta \psi_{1}+\gamma \cos \Delta \psi_{1},
$$

where $\Delta \psi_{0}$ and $\Delta \psi_{1}$ are the angles of rotation of the sections in free and forced oscillations

$$
\Delta \psi_{0}=\theta_{0} \sin (\pi \alpha / L) \sin \left(\omega_{0} t\right), \quad \Delta \psi_{1}=\theta_{1} \sin (\pi \alpha / L) \sin \left(\omega_{0} t\right) .
$$

Using the general rule of superposition of motions [17], we replace the Lagrange variables in the equations for forced oscillations (55) with expressions for the corresponding Euler variables of natural oscillations (54) 


$$
\begin{aligned}
y=\beta \cos \Delta \psi_{1}-\gamma \sin \Delta \psi_{1}= & \left(\beta \cos \Delta \psi_{0}-\gamma \sin \Delta \psi_{0}\right) \cos \Delta \psi_{1}-\left(\beta \sin \Delta \psi_{0}+\gamma \cos \Delta \psi_{0}\right) \sin \Delta \psi_{1}= \\
= & \beta \cos \left(\Delta \psi_{0}+\Delta \psi_{1}\right)-\gamma \sin \left(\Delta \psi_{0}+\Delta \psi_{1}\right) .
\end{aligned}
$$

As a result, we obtain a system of type (34), in which the angle of rotation in the joint oscillation, instead of (38), is equal to the sum of the angles of rotation of the forced and free oscillations

$$
\Delta \psi=\left(\theta_{0}+\theta_{1}\right) \sin (\pi \alpha / L) \sin \left(\omega_{0} t\right) .
$$

The rationale for the energy feasibility of joint oscillations in accordance with equations (40) and (56) differs only slightly from the one given for transverse oscillations. In accordance with equations (40) and (56), particles of rod with volume $\delta V_{0}$, density $\rho_{0}$, and elastic modulus $\kappa$ rotate relative to the $x$-axis with angular velocities

$$
\psi_{t}(\alpha, t)=\left(\theta_{0}+\theta_{1}\right) \omega_{0} \sin (\pi \alpha / L) \cos \left(\omega_{0} t\right)
$$

and due to this, the system acquires kinetic energy

$$
E_{k i n}=\frac{1}{4} \kappa V_{0}(\pi R / L)^{2}\left(\theta_{0}+\theta_{1}\right)^{2} \cos ^{2}\left(\omega_{0} t\right) .
$$

Elastic energy for equations (40) with tensor (2)

$$
x_{i, p}=\left(\begin{array}{ccc}
1 & 0 & 0 \\
-\psi_{\alpha} z & \cos \Delta \psi & -\sin \Delta \psi \\
\psi_{\alpha} y & \sin \Delta \psi & \cos \Delta \psi
\end{array}\right)
$$

considering the invariant (14) is equal to

$$
e_{d e f}=e_{e}+e_{s}=\psi_{\alpha}^{2} r^{2}=\left(\theta_{0}+\theta_{1}\right)^{2} \pi^{2}(r / L)^{2} \cos ^{2}(\pi \alpha / L) \sin ^{2}\left(\omega_{0} t\right) .
$$

The elastic energy in the volume of the rod is

$$
E_{d e f}=\frac{1}{4} \kappa V_{0}(\pi R / L)^{2}\left(\theta_{0}+\theta_{1}\right)^{2} \sin ^{2}\left(\omega_{0} t\right) .
$$

Equations (57), (58) and (53) correspond to harmonic oscillations (40) with changes in angles (56). Kinetic and elastic energies provide a constant value of their sum at any moment

$$
E_{k i n}+E_{d e f}=0,25 \kappa V_{0} \pi^{2}\left(\theta_{0}+\theta_{1}\right)^{2}(R / L)^{2}=\text { const } .
$$

Resonance from the point of view of the law of conservation of energy is possible and allows for a significant increase in the amplitude of the new phase of free oscillation and the energy parameters associated with the amplitude at the expense of internal forces [12].

Structure of local kinematic parameters of the volume energy density (15) - (16), which depend on the average value of its

$$
e=2 / 3+\sqrt{1+\psi_{\alpha}^{2} r^{2}} / 3, \quad e_{e}=3\left(e^{2}-1\right)=\frac{1}{3} \psi_{\alpha}^{2} r^{2}+\frac{4}{3}\left(\sqrt{1+\psi_{\alpha}^{2} r^{2}}-1\right)=e_{e 1}+e_{e 2},
$$


and the standard deviation $e_{s}$

$$
e_{s}=\frac{2}{3} \psi_{\alpha}^{2} r^{2}+\frac{4}{3}\left(1-\sqrt{1+\psi_{\alpha}^{2} r^{2}}\right)=e_{s 1}+e_{s 2},
$$

the same as for transverse vibrations. Considering the series expansion of the function with a square root, we get

$$
\begin{array}{llll}
\frac{e_{e}}{e_{d e f}}=\frac{\psi_{\alpha}^{2} r^{2}}{\psi_{\alpha}^{2} r^{2}}=1, & \frac{e_{e 1}}{e_{d e f}}=\frac{(1 / 3) \psi_{\alpha}^{2} r^{2}}{\psi_{\alpha}^{2} r^{2}}=\frac{1}{3}, & \frac{e_{e 2}}{e_{d e f}}=\frac{(2 / 3) \psi_{\alpha}^{2} r^{2}}{\psi_{\alpha}^{2} r^{2}}=\frac{2}{3}, \\
\frac{e_{s}}{e_{d e f}}=\frac{2}{3}-\frac{2}{3}=0, & \frac{e_{s 1}}{e_{d e f}}=\frac{2}{3} \frac{\psi_{\alpha}^{2} r^{2}}{\psi_{\alpha}^{2} r^{2}}=\frac{2}{3}, & \frac{e_{s 2}}{e_{d e f}}=\frac{(4 / 3)\left(-\psi_{\alpha}^{2} r^{2} / 2\right)}{\psi_{\alpha}^{2} r^{2}}=-\frac{2}{3} .
\end{array}
$$

As in the case of transverse vibrations, the energy fractions $e_{e l}$ and $e_{s l}$ change synchronously and in total provide the elastic energy necessary to fulfill the integral conservation law, which is converted into kinetic energy. The components $e_{e 2}$ and $e_{s 2}$ are 2 times larger than $e_{e l}$, but do not participate in such transformations and determine the energy consumption only for changing the shape of the particles (16), associated with changes in the length of the edges of particles $e_{e}$ and their standard deviation from the mean $e_{s}$. Changes occur with the same frequency, but in opposite phases, energy costs are compensated by the opposite type of deformation.

The analysis shows that part of the energy involved in free and combined vibrations is not associated with energy coming from external sources, is not converted into kinetic energy, but is an integral element of resonant phenomena.

\section{Discussion and conclusions}

The basis of resonance, as well as other physical phenomena, is the law of conservation of energy. The resonance involves two types of energy mentioned in the left-hand sides of equations (38), (59), and (81). They are defined by two invariants of the equations of motion: the square of the velocity (first derivatives of the coordinates in time) and the quadratic invariant (12) of the tensor (2) with the derivatives of the Euler coordinates by Lagrange variables. The experience of using kinetic energy in the mechanics of absolutely solid bodies does not allow us to doubt the correctness of the transition from the square of velocity to the corresponding type of energy.

With elastic energy, the situation is more complicated. There is reason to assume that classical mechanics with three elastic modules does not explain the phenomena associated with resonance, because it does not consider the internal energy involved in the resonance. This energy is considered by the energy model of mechanics with a single elastic modulus with a new scale of average stresses that consider the initial energy state of particles [15]. The conversion of strain (12) to elastic energy according to equation (17) ensures the implementation of the law of conservation of energy both in the volume integral form, considering the transition of part of the elastic energy to kinetic energy, 
and for subsystems with the transformation of deformation-related modifications of the internal elastic energy.

Analysis of the structure of the invariant (12) shows that both external and internal energy sources are used to change the volume or shape of particles in resonance. Changes in the volume of particles in the regions of tension and compression during longitudinal vibrations do not require an influx of energy from outside, just as free vibrations occur in elastic bodies without external additional energy inputs. Similarly, part of the energy for changing the shape of particles during transverse and torsional vibrations comes from internal sources, including the elastic energy of the particles themselves when the deformed state is changed equivalently.

In the mechanics of a deformable solid [10], terms are used about the energies associated with changes in the volume and shape of particles. With transverse and torsional vibrations, the volume of particles does not change, so a new terminology is proposed that clarifies the mathematical meaning of the components of deformation: parts of the energy that are associated either with changes in the average values of the particle edge lengths or with their standard deviations from the average values.

The dimensionless local kinematic parameters of the energy (15) - (16), which differ from the volume density of the corresponding types of elastic energy by a constant factor equal to the elastic modulus of the material, as in equation (13), allow us to identify the features of the energy state of particles under free vibrations and resonance. The nature of the changes during the cycle allows us to judge the possibility of their transformation into kinetic energy with the implementation of the law of conservation of energy for the body as a whole, or only to change the elastic deformation of parts of the body (due to internal sources) without using the energy of external forces. They correspond to real deformations, have a clear geometric interpretation associated with changes in the average relative length of the sides of the particles $\left(e_{e}\right)$ and the standard deviation of these lengths from the average value for each particle $\left(e_{s}\right)$.

Equations (33), (53), (73) they reflect the features of the mechanism for converting forced vibrations with a frequency determined by external influences into their own after the termination of the driving force. This mechanism continues to operate with a superposition of free and forced oscillations, the frequency of which is close to its own, but does not coincide with it.

Equations (33), (53), (73) correspond to the mechanism of transformation of forced vibrations with a frequency determined by external influences into their own after the termination of the driving force. This mechanism continues to operate with a superposition of free and forced oscillations, the frequency of which is close to its own, but does not coincide with it.

Resonance is possible by of a superposition of both similar and different types of vibrations if their frequencies coincide with their own or are close to them [12]. For example, with the superposition of longitudinal and transverse vibrations at the same frequencies that coincide with the 
frequencies of natural vibrations, the volume densities of kinetic and elastic energy, and therefore their volume integral values, have the property of additivity, the law of conservation of energy is fulfilled, and resonance is possible.

The relations (29), (47) and (68) can be used to determine the elastic constant of a material from experimental studies with the main forms of free vibrations.

This research received no external funding. The funders had no role in the design of the study; in the collection, analyses, or interpretation of data; in the writing of the manuscript, or in the decision to publish the results.

Author ID in ORCID http://orcid.org/0000-0001-9977-3342.

\section{References}

1. Timoshenko S. P. Fluctuations in engineering. Nauka, Moscow (1967)

2. Panovko Ya. G. Introduction to the theory of mechanical vibrations. Nauka, Moscow (1991)

3. Panovko Ya. G., Gubanova I. I. Stability and vibrations of elastic systems. Modern concepts, paradoxes and errors. Nauka, Moscow (2007)

4. Biderman V. L. Theory of mechanical vibrations. Higher school, Moscow (1980)

5. Ilyin M. M., Kolesnikov K. S., Saratov Yu. S. Theory of vibrations. MSTU publishing House. Bauman, Moscow (2003)

6. Vibrations in engineering. Vol. 1. Vibrations of linear systems / ed. by V. V. Bolotin. Mashinostroenie, Moscow (1978)

7. Vibration in technology. / Ed. By V. N. Chelomey. Mashinostroenie, Moscow (1979)

8. Alyushin Yu. A. Energy features of free vibrations in elastic bodies. Phisical Mesomech. 22, 3, 77-87 (2019) https://doi.org/10.24411/1683-805X-2018-13007

9. Alyushin $Y u$. A. New Concept in Mechanics Based on the Notions of Space, Time, and Energy. Physical Mesomech, 22(6), 536-546 (2019) https://doi.org/10.1134/S1029959919060109

10. Alyushin Yu. A., Energy Foundations of Mechanics: A Handbook for Students. Mashinostroenie, Moscow (1999)

11. Alyushin Yu. A., Energy fundamentals of mechanics. Lambert Academic Publishing (2016)

12. Alyushin Yu. A., Energy basis of resonance in elastic bodies //. Physical mesomechanics. 22, 5, $42-53$ (2019). https://doi.org/10.24411/1683-805X-2019-15006

13. Aristotle. Essays. Physics. vol. 4. - Moscow: Mir, 1975

14. Alyushin $Y u$. A., Defining Relations of Reversible and Irreversible Deformation in the Lagrangian Description, J. Mashinery Manyfact. Reliabil., 5, 434-442 (2007)

15. Alyushin Yu. A., Energy Scale of the Average Stresses and Physical Properties of metals in the Region of Reversible and Irreversible Deformations, J. Machinery Manufact. Reliabil., 3, 282-289 (2010)

16. Alyushin $Y u$. A. Physical properties of materials that determine reversible and irreversible deformations. Exploratory Material Science Research. 2020 №1.- P. 12-23.

17. Alyushin Yu. A., The Principle of Superposition of Motions in the Space of Lagrangian Coordinares, Probl. Mashinostroen. Nadezhn. Mschin, 3, 13-19 (2001) 